techniques, Hubble discovered that most galaxies seem to recede at a rate proportional to their distance. His 'Hubble constant' quantifies that proportion. Today's state-of-the-art observations suggest that, on average, galaxies' speeds increase by 73.5 kilometres per second for every megaparsec (3.26 million light years) of distance. Thus, for example, galaxies 100 megaparsecs away recede at around 7,350 $\mathrm{km} \mathrm{s}^{-1}$.

This value of the Hubble constant comes from observing stars that act as standard candles. These have known intrinsic brightness, so their distance can be estimated from how bright they look in the sky. But the value of 73.5 clashes with the 66.9 estimated in 2015 by cosmologists who mapped the cosmic microwave background the relic radiation from the Big Bang - using the Planck observatory of the European Space Agency (ESA). The discrepancy could still turn out to be caused by unknown artefacts of the measuring techniques, but both camps say that they are increasingly confident in their results.

The Planck estimate relies on what is known as the standard model of cosmology. It makes assumptions regarding the composition of the Universe, and in particular the content of dark matter and the nature of dark energy, the mysterious driver of the acceleration of the cosmic expansion. So, if the discrepancy holds up, it could point to entirely new physics, implying that dark matter is stranger than physicists had assumed, or that the effects of dark energy change with time.

By contrast, some wonder whether standard candles might not be as reliable as astronomers think. This month, another ESA mission, the Gaia telescope, will release a 3D map of the Milky Way that has unprecedented precision and depth, and will help astronomers test the reliability of these cosmic signposts. But, ideally, astronomers would like to have more direct ways of measuring distances outside our Galaxy.

Enter gravitational waves. These stand ready to address some classic astronomical challenges with strong new evidence, as described in a News Feature on page 164. They might also help to resolve the issues surrounding the cosmic expansion. Health warning: these possibilities are speculative and controversial.

When two cosmic orbs - such as the neutron stars seen merging last August - spiral into each other, they emit gravitational waves that carry information about their distance, constituting a 'standard siren'. This enabled physicists at the US-based Laser Interferometer Gravitational-Wave Observatory (LIGO) to calculate the Hubble constant.

"If the
discrepancy
holds up, it
could point to
entirely new
physics."
They obtained a value of 70 , smack in the middle of the standard-candle and cosmicmicrowave-background estimates. LIGO's data point has a large margin of error, but, as researchers collect more of these events, the results might end up leaning conclusively one way or the other.

Ultimately, gravitational waves could enable researchers to measure not just the current cosmic expansion, but also how the rate of expansion has evolved over the aeons. Two upcoming ESA projects will help enormously, especially if they get to fly at the same time, as many researchers hope. The gravitational-wave detector LISA (Laser Interferometer Space Antenna) should detect mergers of black holes across the Universe's history. And some astronomers anticipate that the X-ray observatory Athena (Advanced Telescope for High-Energy Astrophysics) might pick up photons from the same events and help researchers find the corresponding galaxies' redshifts - although others consider this a long shot.

Mapping standard sirens in this way should shed light on the nature of dark energy - cosmologists' most coveted goal. They hope that it will provide hints about the future of the Universe. Predictions for an infinitely long-lasting future are outside the realm of science. But cosmologists could still work out whether cosmic expansion will continue to accelerate for the foreseeable future, or whether that acceleration might increase, stop or perhaps reverse.
ANNOUNCEMENT

\section{Awards to celebrate women in science}

$\mathrm{F}_{\mathrm{h}}^{\mathrm{e}}$ male scientists are under-represented in global research. Nature has long argued the need for initiatives to increase their opportunities and participation - so we are delighted to announce an awards programme that aims to do both.

The two annual awards will recognize inspirational early-career female researchers and those who have worked to champion young women's and girls' participation in science. By rewarding and celebrating these achievements, we hope the programmes will contribute to a positive shift towards the equity sorely needed in the research community.

The first is called the Inspiring Science Award and will honour female scientists who have completed their $\mathrm{PhD}$ within the past ten years and have made an exceptional contribution to scientific discovery, as reflected in publications, poster and conference presentations, leadership, tutoring and mentoring. Candidates can be nominated by anyone in their research institute, and we encourage nominations from around the globe and across all subject areas. Our independent judging process will ensure that those working under adverse circumstances or in regions where there is limited access to scientific literature will not be unfairly disadvantaged.

The second prize, the Innovating Science Award, recognizes individuals or organizations that have led a grass-roots initiative to support increased access to, or interest in, science, technology, engineering and mathematics (STEM) for girls and young women around the globe. This backs our belief that supporting early interest in STEM worldwide is a crucial step towards sustainably increasing the representation of women in these subjects. Candidates for this award can nominate themselves.

Nominations opened on 9 April and will close on 11 June 2018. A longlist of ten nominees for each award will be announced on 24 July, and a shortlist of five will be announced on 4 September. Both awards are run by Nature Research in partnership with The Estée Lauder Companies. (Full details of the criteria and nomination processes are available at nature.com/researchawards.)

The winners of the awards will be announced in October. They will receive grants of US\$10,000 to build on their efforts, and an invitation to an award ceremony. The Inspiring Science Award winner will also receive a grant of up to $\$ 5,200$ to support open-access publication of their research, and the Innovating Science Award winner will receive up to $\$ 5,200$ to support an event that showcases their initiative. These awards complement the existing Nature Awards for Mentoring in Science and the John Maddox Prize for promoting sound science and evidence on a matter of public interest.

Nature strives to champion and showcase the achievements of researchers, and we have a responsibility to drive positive change in the research community. Our journals are committed to supporting gender equity (see go.nature.com/2glxtdj for a collection of related content). We recognize that a huge amount must be done to overcome the many barriers that women face to entry and progression in research; these awards are just one small contribution. We look forward to identifying outstanding individuals who are deserving of these awards, celebrating their achievements and sharing their stories. 